\title{
Classical solutions to a moving boundary problem for an elliptic-parabolic system
}

\author{
JOACHIM ESCHER ${ }^{\dagger}$ \\ Institute for Applied Mathematics, University of Hannover, Hannover, Germany
}

[Received 6 September 2002 and in revised form 17 December 2003]

\begin{abstract}
The paper concerns a moving boundary problem for a coupled system of an elliptic and a parabolic boundary value problem. This system is applied to a model describing the growth of a homogeneous solid tumor in which the cell proliferation rate depends on the nutrient concentration only. For a large class of initial data the existence of a unique classical solution is shown.
\end{abstract}

\section{Introduction}

In this paper we investigate a simple model describing in vivo cancer growth for a single tumor. The model comprises a reaction-diffusion equation describing the evolution of the nutrient concentration, denoted by $u$, and an elliptic equation for the internal pressure, denoted by $p$, in the tissue. The cell proliferation rate is denoted by $f(u)$, where $f: \mathbb{R} \rightarrow \mathbb{R}$ is assumed to be smooth. Typically, this proliferation rate $f$ is assumed to be linear or of logistic type (cf. [6, p. 157] and [7. pp. 190, 193]). A polynomial proliferation rate is proposed in the Appendix of [16]. At time $t$ the tumor occupies the domain $\Omega(t)$ with the moving boundary $\Gamma(t)$. In dimensionless form $p$ and $u$ satisfy the equations

$$
\begin{aligned}
-\Delta p & =f(u) & & \text { in } \Omega(t), \\
\partial_{t} u-\Delta u+u & =0 & & \text { in } \Omega(t) .
\end{aligned}
$$

To formulate the boundary conditions satisfied by $p$ and $u$, let $V$ denote the normal velocity of the boundary, i.e. the component of the derivative of $t \mapsto \Gamma(t)$ in direction of the outward normal $v(t)$. Moreover, we write $H(t)$ for the mean curvature of $\Gamma(t)$ and $\psi$ for the nutrient concentration outside the tumor. Then we have the following boundary conditions:

$$
\begin{array}{ll}
V=-\partial_{\nu} p & \text { on } \Gamma(t), \\
p=c H & \text { on } \Gamma(t), \\
u=\psi & \text { on } \Gamma(t) .
\end{array}
$$

Here $c$ is a positive constant. Further we use the sign convention that $V$ is positive if the tumor grows and that $H$ is positive if $\Gamma(t)$ is convex with respect to $\Omega(t)$. Finally, we close the system by the initial conditions

$$
\Gamma(0)=\Gamma_{0}, \quad u(0, \cdot)=u_{0},
$$

where $\Omega_{0}$ denotes the domain initially occupied by the tumor, so that $\Gamma_{0}:=\partial \Omega_{0}$ is the initial shape of the tumor. The initial nutrient concentration is denoted by $u_{0}$. Finally, we assume that

†Email: escher@ifam.uni-hannover.de 
$\psi: \mathbb{R}^{n} \rightarrow \mathbb{R}$ is smooth and bounded and has bounded derivatives of arbitrary order. We mention that the case $\psi \equiv c$ for some $c \in \mathbb{R}$ is of particular interest in applications. In order to state our main result we denote the usual Sobolev and Besov spaces by $W_{q}^{k}$ and $B_{q q}^{s}$, respectively, where $q \in[1, \infty], k \in \mathbb{N}$, and $s \geqslant 0$ (cf. [21]).

THEOREM 1 Let $n \geqslant 2$ and $q>n+1$. Let further $\Omega_{0}$ be a bounded domain in $\mathbb{R}^{n}$ of class $B_{q q}^{4-1 / q}$ and assume that $u_{0}-\psi \in W_{q, 0}^{2}\left(\Omega_{0}\right)$. Then system (1.1)-(1.6) has a unique classical solution $(p, u, \Gamma)$ of class $W_{q}^{2} \times W_{q}^{2} \times B_{q q}^{4-1 / q}$ on some time interval $[0, T)$ with $T>0$. This solution is smooth on the time-space manifold

$$
\bigcup_{t \in(0, T)}(\{t\} \times \bar{\Omega}(t))
$$

We refer to Sections 3 and 4 for the proof of this result. The precise spatial and temporal regularity of the solution as well as the class in which the solution is unique can be found there too.

Let $\Gamma_{0}$ be a compact hypersurface of $\mathbb{R}^{n}$ which is of class $C^{4}$. Then it follows from Proposition 2.3.2 of [21] that $\Gamma_{0}$ is of class $B_{q q}^{4-1 / q}$. Thus any bounded $C^{4}$-domain in $\mathbb{R}^{n}$ is within the scope of Theorem 1.

Let us briefly exhibit the method used to prove the above result. In the first step we introduce suitable diffeomorphisms to transform the original domains $\Omega(t)$ and original moving boundary $\Gamma(t)$ onto a fixed reference domain $D$ with a fixed boundary $\Sigma$, respectively. Next we perform a reduction of the transformed system by eliminating the pressure $p$. We are then left with a nonlinear system of the form

$$
\partial_{t} X=\mathbb{F}(X), \quad X(0)=X_{0},
$$

where $X$ and $X_{0}$ denote the transformed pairs $(u, \Gamma)$ and $\left(u_{0}, \Gamma_{0}\right)$, respectively. Besides the transformed differential operators from (1.2) and (1.3) the operator $\mathbb{F}$ contains the solution operator for the (transformed) pressure $p$ as well. It turns out that $\mathbb{F}$ is a quasilinear and nonlocal operator. However, we show in Section 4 that the evolution equation is of parabolic type, in the sense that $\mathbb{F}$ contains a leading linear term which generates an analytic semigroup on a suitable product of Sobolev spaces over $D$ and Besov spaces over $\Sigma$. Using the notation of Section 4 , we have $\mathbb{F}(Z)=$ $-\mathbb{A}(Z) Z+F(Z)$, with the quasilinear parabolic leading term $\mathbb{A}$ and the lower order term $F$. Some technical effort is needed to establish appropriate mapping properties of the nonlinear operators $Z \mapsto F(Z)$ and $Z \mapsto \mathbb{A}(Z)$ and the generation property of the linear operator $Z \mapsto-\mathbb{A}\left(Z_{0}\right) Z$ with $Z_{0}$ fixed. This is done in the first part of Section 4. In the second part we prove the main result by solving the quasilinear evolution equation

$$
Z^{\prime}+\mathbb{A}(Z) Z=F(Z), \quad Z(0)=Z_{0} .
$$

Here we rely on the well established theory of quasilinear parabolic evolution equations which is based on analytic semigroups (see [2] or [20]).

Roughly speaking, moving boundary problems may be regarded as nonlinear evolution equations for the boundary manifolds (in the present case this evolution equation is coupled to the parabolic equation for the nutrient concentration $u$ ). Mostly, these evolution equations are of quasilinear, sometimes even of fully nonlinear type. Therefore, any loss of regularity in the construction of classical solutions shatters the possibility to go beyond a mere existence result (for classical solutions). It is worth mentioning that the functional analytic frame proposed here avoids 
any loss of regularity and offers therefore an excellent basis to study further properties of solutions to the system (1.1)-(1.6). Of particular interest are certainly blow-up behaviour or global existence of solutions. It is for instance possible to show that a solution exists globally, provided the nutrient concentration $u$ is a priori bounded in $W_{q}^{1}$ and the moving boundary is a priori bounded in $B_{q q}^{5 / 2-1 / q}$. This result as well as further dynamical properties of the system (1.1)-(1.6) will be the subject of a forthcoming paper.

In order to evade a loss of regularity, one first needs a suitable tool to measure precisely the boundary regularity of functions on a domain. This is obvious for (Hölder-)continuous functions. In the case of Sobolev spaces $W_{q}^{k}$ (which is a widely used class of function spaces to treat parabolic problems), the boundary regularity is characterized by means of Besov spaces: Given $u \in W_{q}^{k}(\Omega)$ with $k \geqslant 1, q \in(1, \infty)$ and $\partial \Omega$ smooth, the restriction $u \mid \partial \Omega$ of $u$ to the boundary $\partial \Omega$ belongs to the Besov space $B_{q q}^{k-1 / q}(\partial \Omega)$; and vice versa: given any $\varphi \in B_{q q}^{k-1 / q}(\partial \Omega)$ there is a $u \in W_{q}^{k}(\Omega)$ such that $u \mid \partial \Omega=\varphi$. Consequently, if one prefers to solve the parabolic equation for $u$ in some Sobolev spaces $W_{q}^{k}(\Omega)$, the right choice of the function spaces to solve for the moving boundary is a suitable Besov space.

System (1.1)-(1.6) was introduced by Greenspan in [16] and [17], see also [7]. Mostly the radially symmetric situation was investigated in a recent series of papers by Friedman and Reitich [13, 14, 15]. Existence and uniqueness of nonsymmetric solutions have been shown even more recently in [5] in the physically relevant case of space dimension $n=3$. However, existence of solutions is guaranteed in [5] only under high regularity assumptions on the initial data, and a serious loss of regularity of the solution could not be excluded. More precisely, it is shown in the main result of [5] that, given $\Omega_{0}$ of class $C^{17+\alpha}$ and $u_{0}$ of class $C^{13+\alpha}$, the solution $(u, \Gamma)$ belongs to $C^{7+\alpha} \times C^{11+\alpha}$. For domains of dimension higher than 3 the regularity of the initial value in [5] has even to be increased.

We emphasize that the regularity properties of the solution constructed by the above Theorem 1 are optimal with respect to that point (see Section 4). Moreover, we show that there is not only no loss of regularity of the solutions during the evolution but even a strong regularizing effect: For each positive time the solutions are smooth in time and in space up to the boundary. Finally, we mention that an analytic framework in which a loss of regularity of solutions cannot be excluded is not appropriate to deduce any global existence or blow-up results. Taking e.g. the framework of [5] one has to bound a priori the moving boundary in $C^{17+\alpha}$ and the nutrient concentration in $C^{13+\alpha}$ in order to guarantee that a solution exists globally.

Assume that the proliferation rate $f$ vanishes. Then the original moving boundary problem (1.1)-(1.6) reduces to the one phase Mullins-Sekerka problem

$$
\Delta p=0 \quad \text { in } \Omega(t), \quad V=-\partial_{\nu} p \quad \text { and } \quad p=c H \quad \text { on } \Gamma(t)
$$

for the pressure $p$ and the moving boundary $\Gamma$ (cf. [4, 8, 11, 12]). However, if $f$ is nontrivial, the results obtained in these papers cannot be used to solve (1.1)-(1.6) for the following reason: The second equation of (2.15) below shows that, even in the case of a linear proliferation rate $f(u)=-\mu(u-\bar{u})$ (with positive constants $\mu$ and $\bar{u}$ ), there is a nonlinear coupling of $p, u$, and $\Gamma$. Since the methods in [4, 8, 11, 12] are designed to solve a quasi-stationary problem, the temporal regularity of $p$ is not sufficient to go successfully through a fixed point iteration for $u$ coming from a parabolic equation (although the spatial regularity of $p$ obtained in [4, 8, 11, 12] is optimal). Therefore we propose here a different approach: We first eliminate the pressure $p$ from the system and then solve the reduced system simultaneously for $(u, \Gamma)$. 


\section{The transformed system}

Let $\Omega_{0}$ be a bounded domain in $\mathbb{R}^{n}$ such that its boundary $\Gamma_{0}$ is a compact orientable hypersurface of class $C^{3}$. Then in any tubular neighbourhood of $\Gamma_{0}$ there is a smooth hypersurface $\Sigma$ (see Section 8 in [19]). Let $\mu$ be the outward unit normal field on $\Sigma$. Then for $a>0$ small enough, the mapping

$$
X: \Sigma \times(-a, a) \rightarrow \mathbb{R}^{n}, \quad(p, r) \mapsto p+r \mu(p),
$$

is a smooth diffeomorphism onto its range $\mathcal{R}:=\operatorname{im}(X)$, i.e. $X \in \operatorname{Diff}^{\infty}(\Sigma \times(-a, a), \mathcal{R})$. We further assume that $\Gamma_{0} \subset \mathcal{R}$. If $\Gamma_{0}$ is smooth this assumption obviously holds true by choosing $\Sigma=\Gamma_{0}$.

Later on it will be convenient to decompose the inverse of $X$ as $X^{-1}=(P, \Lambda)$ such that

$$
P \in C^{\infty}(\mathcal{R}, \Sigma), \quad \Lambda \in C^{\infty}(\mathcal{R},(-a, a)) .
$$

Here $P$ is the metric projection of $\mathcal{R}$ onto $\Sigma$, i.e. $P(x)$ is the nearest point on $\Sigma$ to $x$, and $\Lambda(x)$ is the signed distance from $x$ to $\Sigma$. Clearly, $\mathcal{R}$ is the set of those points with distance less than $a$ to $\Sigma$. For $b \in(0, a)$, we call

$$
\operatorname{Ad}:=\operatorname{Ad}_{b}:=\left\{\rho \in C^{2}(\Sigma) ;\|\rho\|_{C^{1}(\Sigma)}<b\right\}
$$

the set of admissible functions. Given $\rho \in \mathrm{Ad}$, let

$$
\theta_{\rho}: \Sigma \rightarrow \mathbb{R}^{n}, \quad p \mapsto p+\rho(p) \mu(p),
$$

and $\Gamma_{\rho}:=\operatorname{im}\left(\theta_{\rho}\right)$. Then $\Gamma_{\rho}$ is a $C^{2}$-hypersurface which is diffeomorphic to $\Sigma$, i.e. $\theta_{\rho} \in$ $\operatorname{Diff}^{2}\left(\Sigma, \Gamma_{\rho}\right)$. Moreover, there is a $\rho_{0} \in$ Ad such that $\Gamma_{\rho_{0}}=\Gamma_{0}$, since $\Gamma_{0} \subset \mathcal{R}$. We write $\Omega_{\rho}$ for the domain enclosed by $\Gamma_{\rho}$. Clearly, we have $\Omega_{\rho_{0}}=\Omega_{0}$. For simplicity we also set $D:=\Omega_{\rho \equiv 0}$, which will be our fixed reference domain. Observe that $\Sigma$ is the boundary of $D$.

We further need a suitable extension $\Theta_{\rho}: \mathbb{R}^{n} \rightarrow \mathbb{R}^{n}$ of $\theta_{\rho}$ to $\mathbb{R}^{n}$. To this end we introduce a special case of the so-called Hanzawa transformation. Let $b \in(0, a / 4)$ be given, and pick $\varphi \in$ $C^{\infty}(\mathbb{R},[0,1])$ such that

$$
\varphi(r)=\left\{\begin{array}{ll}
1 & \text { if }|r| \leqslant b, \\
0 & \text { if }|r|>3 b,
\end{array} \text { and } \quad \sup \left|\varphi^{\prime}(r)\right|<1 / b .\right.
$$

Then, given $\rho \in \mathrm{Ad}$, define

$$
\Theta_{\rho}(x):= \begin{cases}X(P(x), \Lambda(x)+\varphi(\Lambda(x)) \rho(P(x))) & \text { if } x \in \mathcal{R}, \\ x & \text { if } x \notin \mathcal{R} .\end{cases}
$$

Observe that $\left|\varphi^{\prime}(r) \rho(p)\right|<1$ for $\rho \in \operatorname{Ad}$ and $r \in \mathbb{R}, p \in \Sigma$. Hence the function $r \mapsto r+\varphi(r) \rho(p)$ is strictly increasing for any $\rho \in$ Ad. From this one easily deduces that $\Theta_{\rho} \in \operatorname{Diff}^{2}\left(\mathbb{R}^{n}, \mathbb{R}^{n}\right)$ with

$$
\Theta_{\rho} \in \operatorname{Diff}^{2}\left(D, \Omega_{\rho}\right), \quad \Theta_{\rho} \mid \Sigma=\theta_{\rho} .
$$

We now consider time-dependent functions $\rho: J \rightarrow$ Ad, where $J:=[0, T]$ for some $T>0$. More precisely, given

$$
\rho \in C^{1}(J, C(\Sigma)) \cap C(J, \mathrm{Ad})
$$


we obtain a family

$$
\Gamma:=\left\{\Gamma(t)=\Gamma_{\rho(t)} ; t \in J\right\}
$$

of hypersurfaces in $\mathbb{R}^{n}$. The domains enclosed by those hypersurfaces are denoted by $\Omega(t)=$ $\Omega_{\rho(t)}, t \in J$. It will be convenient to describe $\Gamma(t)$ as the zero-level set of a suitable time-dependent function. Letting

$$
\phi_{\rho}: J \times \mathcal{R} \rightarrow \mathbb{R}, \quad(t, x) \mapsto \Lambda(x)-\rho(t, P(x)),
$$

we obviously have $\Gamma(t)=\phi_{\rho}(t, \cdot)^{-1}(0)$. Hence the outward unit normal field $v(t, \cdot)$ on $\Gamma(t)$ at the point $x=X(s, \rho(t, s))$ can be represented as

$$
v(t, s)=\left.\frac{\nabla \phi_{\rho}(t, x)}{\left|\nabla \phi_{\rho}(t, x)\right|}\right|_{x=X(s, \rho(t, s))} \quad \text { for }(t, s) \in J \times \Sigma .
$$

In order to calculate the normal velocity of $t \mapsto \Gamma(t)$ at time $t \in[0, T)$ and at the point $x=$ $X(s, \rho(t, s))$, let us introduce the auxiliary function $\Psi$ by setting

$$
\Psi(\tau, r):=\phi_{\rho}(t+\tau, x+r v(t, s))=\Lambda(x+r v(t, s))-\rho(t+\tau, P(x+r v(t, s)))
$$

for $(\tau, r) \in[0, T-t) \times[0, a-b)$. Clearly $\Psi(0,0)=0$ and

$$
\partial_{2} \Psi(0,0)=\nabla \phi_{\rho}(t, x) \cdot v(t, s)=\left|\nabla \phi_{\rho}(t, x)\right| \neq 0 .
$$

Hence the implicit function theorem implies that there is an $\varepsilon>0$ and a function $h \in C^{1}([0, \varepsilon), \mathbb{R})$ such that $\Psi(\tau, h(\tau))=0$ for $\tau \in[0, \varepsilon)$ with

$$
h^{\prime}(0)=\frac{-\partial_{1} \Psi(0,0)}{\partial_{2} \Psi(0,0)}=\frac{\partial_{t} \rho(t, s)}{\left|\nabla \phi_{\rho}(t, x)\right|} .
$$

Observe that $x+h(\tau) v(t, s)$ belongs to $\Gamma(t+\tau)$. Thus $h(\tau)$ is the increase at $x=X(s, \rho(t, s)) \in$ $\Gamma(t)$ in the direction of the outer normal $v(t, s)$ so that the normal velocity $V(t, s)$ of $t \mapsto \Gamma(t)$ at $t$ and $x=X(s, \rho(t, s))$ is given by $h^{\prime}(0)$. Therefore (2.6) yields

$$
V(t, s)=\frac{\partial_{t} \rho(t, s)}{\left|\nabla \phi_{\rho}(t, x)\right|_{x=X(s, \rho(t, s))}}, \quad(t, s) \in J \times \Sigma .
$$

We can now rewrite the one-phase Stefan condition (1.3) in the form

$$
\partial_{t} \rho(t, s)=-\left.\left(\nabla p(t, x) \mid \nabla \phi_{\rho}(t, x)\right)\right|_{x=X(s, \rho(t, s)), \quad(t, s) \in J \times \Sigma,}
$$

where $(\cdot \cdot \cdot)$ stands for the Euclidean inner product in $\mathbb{R}^{n}$.

We need some further notation. Let

$$
\Omega_{\rho, J}:=\bigcup_{t \in J}\left(\{t\} \times \Omega_{\rho(t)}\right) \quad \text { for } \rho \in C^{1}(J, C(\Sigma)) \cap C(J, \mathrm{Ad})
$$

and define

$$
\Phi_{\rho}: J \times D \rightarrow \Omega_{\rho, J}, \quad(t, y) \mapsto\left(t, \Theta_{\rho(t)}(y)\right) .
$$

Then $\Phi_{\rho} \in \operatorname{Diff}^{0}\left(J \times D, \Omega_{\rho, J}\right)$ and hence the pull-back operator $\Phi_{\rho}^{*}$, defined by

$$
\Phi_{\rho}^{*} u:=u \circ \Phi_{\rho} \quad \text { for } \quad u \in C\left(\Omega_{\rho, J}\right),
$$


as well as the push-forward operator $\Phi_{*}^{\rho}$, given by

$$
\Phi_{*}^{\rho} v:=v \circ \Phi_{\rho}^{-1} \quad \text { for } v \in C(J \times D),
$$

are well defined. Using this notation we introduce the following transformed differential operators:

$$
A(\rho) w:=-\Phi_{\rho}^{*} \Delta\left(\Phi_{*}^{\rho} w\right), \quad B(\rho) w:=\Phi_{\rho}^{*}\left(\nabla\left(\Phi_{*}^{\rho} w\right) \mid \nabla \phi_{\rho}\right)
$$

for $w \in C\left(J, W_{p}^{2}(D)\right)$. Of course we also need the transformed versions of the nonlinear operator $f$ and the mean curvature, respectively:

$$
g(w, \rho):=\Phi_{\rho}^{*} f\left(\Phi_{*}^{\rho} w\right), \quad H(\rho):=\Phi_{\rho}^{*} H_{\Gamma_{\rho}},
$$

where $H_{\Gamma_{\rho(t)}}$ denotes the mean curvature of $\Gamma_{\rho(t)}$ for $t \in J$.

Furthermore, given

$$
w \in C\left(J, C^{1}(D)\right), \quad q \in C\left(J, C^{1}(D)\right), \quad \rho \in C(J, \mathrm{Ad}),
$$

we introduce a mapping $R(w, q, \rho)$ on $D$ by

$$
R(w, q, \rho)(y):= \begin{cases}{[\varphi \circ \Lambda] \cdot[B(\rho) q \circ P] \cdot\left[B_{\mu}(\rho) w\right](y),} & y \in \mathcal{R}_{D}, \\ 0, & y \in D \backslash \mathcal{R}_{D},\end{cases}
$$

where $B_{\mu}(\rho) w:=\left(\Phi_{\rho}^{*}\left(\nabla \Phi_{*}^{\rho} w\right) \mid \mu \circ P\right)$ and $\mathcal{R}_{D}:=\mathcal{R} \cap D$. The transformed boundary initial data are

$$
\chi(\rho):=\Phi_{\rho}^{*} \psi, \quad w_{0}:=\Theta_{\rho_{0}}^{*} u_{0} .
$$

Then it follows from (2.11)-(2.14) that the original system (1.1)-(1.6) can be transformed into the following system for $r:=\Phi_{\rho}^{*} p$ and $w:=\Phi_{\rho}^{*} u$ :

$$
\begin{aligned}
A(\rho) r & =g(w, \rho) & & \text { in } J \times D, \\
\partial_{t} w+A(\rho) w & =R(w, r, \rho)-w & & \text { in } J \times D, \\
\partial_{t} \rho+B(\rho) r & =0 & & \text { on } J \times \Sigma, \\
r & =H(\rho) & & \text { on } J \times \Sigma, \\
w & =\chi(\rho) & & \text { on } J \times \Sigma, \\
w(0, \cdot) & =w_{0} & & \text { in } D, \\
\rho(0, \cdot) & =\rho_{0} & & \text { in } \Sigma .
\end{aligned}
$$

Let now $\rho_{0} \in B_{q q}^{4-1 / q}(\Sigma) \cap$ Ad and $w_{0} \in W_{q}^{2}(D)$ with $q \in(1, \infty)$ be given. We call $(w, r, \rho)$ a classical solution to $(2.15)$ with initial data $\left(w_{0}, \rho_{0}\right)$ if the following conditions hold:

(i) $\rho \in C\left(J, B_{q q}^{4-1 / q}(\Sigma) \cap \mathrm{Ad}\right) \cap C^{1}\left(J, B_{q q}^{1-1 / q}(\Sigma)\right) \cap C^{\infty}\left(\dot{J}, B U C^{\infty}(\Sigma)\right)$;

(ii) $w \in C^{\infty}(\dot{J} \times D, \mathbb{R})$;

(iii) $(w, r) \in C\left(J, W_{q}^{2}(D) \times W_{q}^{2}(D)\right)$;

(iv) $(w, r, \rho)$ satisfies $(2.15)$ pointwise on $J \times \bar{D}$.

In order to give a precise notion of a classical solution to (1.1)-(1.6) we assume that

$$
\left\{\begin{array}{l}
\bullet \Gamma_{0} \text { satisfies the assumption stated at the beginning of this section; } \\
\bullet u_{0} \in W_{q}^{2}\left(\Omega_{0}\right) \\
\bullet \psi \in B U C^{\infty}\left(\mathbb{R}^{n}, \mathbb{R}\right) .
\end{array}\right.
$$


Here $B U C^{\infty}$ stands for the Fréchet space consisting of all bounded smooth functions which have bounded and uniformly continuous derivatives of arbitrary order.

Given $\rho \in C^{1}(J, C(\Sigma)) \cap C(J$, Ad), let

$$
\Omega_{\rho, j}:=\bigcup_{t \in(0, T)}\left(\{t\} \times \Omega_{\rho(t)}\right)
$$

and observe that $\Omega_{\rho, j}$ is an open subset of $\mathbb{R}^{n+1}$. We call $(u, p, \Gamma)$ a classical solution to (1.1)-(1.6) with initial data $\left(u_{0}, \Gamma_{0}\right)$ and boundary value $\psi$ if there exists a $\rho \in C^{1}(J, C(\Sigma)) \cap C(J$, Ad) such that the following conditions hold:

(i) $\Gamma(t)=\Gamma_{\rho(t)}$ for $t \in J$;

(ii) $u \in C^{\infty}\left(\Omega_{\rho, j}, \mathbb{R}\right)$;

(iii) $(u(t, \cdot), p(t, \cdot)) \in W_{q}^{2}\left(\Omega_{\rho(t)}\right) \times W_{q}^{2}\left(\Omega_{\rho(t)}\right)$ for $t \in J$;

(iv) $(u, p, \Gamma)$ satisfies $(1.1)-(1.6)$ pointwise on $\bigcup_{t \in J}\left(\{t\} \times \bar{\Omega}_{\rho(t)}\right)$.

LEMma 2.1 Let $\rho_{0} \in B_{q q}^{4-1 / q}(\Sigma) \cap$ Ad be such that $\Gamma_{0}=\Gamma_{\rho_{0}}$ and let $w_{0}:=\Theta_{\rho_{0}}^{*} u_{0}$. Moreover assume that $(w, r, \rho)$ is a classical solution to $(2.15)$ with initial data $\left(w_{0}, \rho_{0}\right)$ and define

$$
u:=\Phi_{*}^{\rho} w, \quad p:=\Phi_{*}^{\rho} r, \quad \Gamma(t):=\Gamma_{\rho(t)}, \quad t \in J .
$$

Then $(u, p, \Gamma)$ is a classical solution to (1.1)-(1.6) with initial data $\left(u_{0}, \Gamma_{0}\right)$ and boundary value $\psi$.

Proof. (i) It is clear that $\Gamma(t)=\Gamma_{\rho(t)}, t \in J$, satisfies the first requirement on $(u, p, \Gamma)$ to be a classical solution to (1.1)-(1.6).

(ii) Let us next verify the regularity of $u$. For this pick $\left(t_{0}, x_{0}\right) \in \Omega_{\rho, j}$. Then $t_{0}>0$ and $x_{0} \in \Omega_{\rho\left(t_{0}\right)}$.

(a) By assumption, $\rho\left(t_{0}\right)$ belongs to $B U C^{\infty}(\Sigma)$. This implies that $\Gamma_{\rho\left(t_{0}\right)}$ is of class $C^{\infty}$ and $\Theta_{\rho\left(t_{0}\right)} \in \operatorname{Diff}^{\infty}\left(D, \Omega_{\rho\left(t_{0}\right)}\right)$. Furthermore, again by assumption, $w\left(t_{0}, \cdot\right) \in C^{\infty}(D, \mathbb{R})$. Hence

$$
u\left(t_{0}, \cdot\right)=w\left(t_{0}, \cdot\right) \circ \Theta_{\rho\left(t_{0}\right)}^{-1} \in C^{\infty}\left(\Omega_{\rho\left(t_{0}\right)}, \mathbb{R}\right) .
$$

(b) If $x_{0}$ does not belong to $\mathcal{R}$ we have $\Theta_{\rho(t)}^{-1}\left(x_{0}\right)=x_{0}$ for all $t \in \dot{J}$. Consequently, $u\left(t, x_{0}\right)=$ $w\left(t, x_{0}\right)$ for all $t \in \dot{J}$, showing that $u\left(\cdot, x_{0}\right) \in C^{\infty}(\dot{J}, \mathbb{R})$.

(c) Assume now that $x_{0} \in \mathcal{R}$, and set $y_{0}:=\Theta_{\rho\left(t_{0}\right)}^{-1}\left(x_{0}\right) \in D$. Furthermore, given $(t, y) \in$ $\dot{J} \times \mathcal{R}_{D}$, define the auxiliary function

$$
\hat{H}(t, y):=P(y)+[\Lambda(y)+\varphi(\Lambda(y)) \rho(t, P(y))] \mu(P(y))-x_{0} .
$$

By assumption, $\rho \in C^{\infty}(\dot{J} \times \Sigma, \mathbb{R})$ and hence (2.1) implies that $\hat{H} \in C^{\infty}\left(\dot{J} \times \mathcal{R}_{D}, \mathbb{R}\right)$. Observe that

$$
\hat{H}(t, y)=\Theta_{\rho(t)}(y)-x_{0}, \quad(t, y) \in \dot{J} \times \mathcal{R}_{D} .
$$

Consequently, $\hat{H}\left(t_{0}, y_{0}\right)=0$ and

$$
\partial_{2} \hat{H}\left(t_{0}, y_{0}\right)=D \Theta_{\rho\left(t_{0}\right)}\left(y_{0}\right) \in \mathcal{G} \mathcal{L}\left(\mathbb{R}^{n}\right) .
$$


Therefore, the implicit function theorem guarantees the existence of an $\varepsilon>0$ and a unique $\pi \in$ $C^{\infty}\left(I_{\varepsilon}, \mathcal{R}_{D}\right)$, where $I_{\varepsilon}:=\left(t_{0}-\varepsilon, t_{0}+\varepsilon\right)$, such that $\hat{H}(t, \pi(t))=0$ for $t \in I_{\varepsilon}$. This implies that

$$
\pi(t)=\Theta_{\rho(t)}^{-1}\left(x_{0}\right) \in \mathcal{R}_{D} \quad \text { for } t \in I_{\varepsilon},
$$

and that

$$
\pi^{\prime}(t)=-\varphi(\Lambda(\pi(t))) \partial_{t} \rho(t, P(\pi(t)))\left[D \Theta_{\rho(t)}(\pi(t))\right]^{-1} \mu(P(\pi(t)))
$$

for $t \in I_{\varepsilon}$. Observe now that

$$
u\left(t, x_{0}\right)=\Phi_{*}^{\rho} w\left(t, x_{0}\right)=w\left(t, \Theta_{\rho(t)}^{-1}\left(x_{0}\right)\right)=w(t, \pi(t)), \quad t \in I_{\varepsilon} .
$$

In particular, we see that $u\left(\cdot, x_{0}\right) \in C^{\infty}\left(I_{\varepsilon}, \mathbb{R}\right)$. In view of (2.17) this shows that $u \in C^{\infty}\left(\Omega_{\rho, j}, \mathbb{R}\right)$.

(iii) Recall that $\rho \in C\left(J, B_{q q}^{4-1 / q}(\Sigma) \cap \mathrm{Ad}\right)$. This implies that

$$
\Theta_{*}^{\rho(t)} \in \operatorname{Isom}\left(W_{q}^{2}(D), W_{q}^{2}\left(\Omega_{\rho(t)}\right)\right), \quad t \in J .
$$

Since $(w(t), r(t)) \in W_{q}^{2}(D) \times W_{q}^{2}(D)$, we conclude that

$$
(u(t, \cdot), p(t, \cdot))=\left(\Theta_{*}^{\rho(t)} w(t), \Theta_{*}^{\rho(t)} r(t)\right) \in W_{q}^{2}\left(\Omega_{\rho(t)}\right) \times W_{q}^{2}\left(\Omega_{\rho(t)}\right) .
$$

(iv) With the notation from (ii), we obtain

$$
\partial_{t} u\left(t, x_{0}\right)=w^{\prime}(t)(\pi(t))+\left(\nabla_{y} w(t)(\pi(t)) \mid \pi^{\prime}(t)\right), \quad t \in I_{\varepsilon} .
$$

Using further the equation $\partial_{t} \rho(t, s)-B(\rho(t)) r(t, s)=0$ on $J \times \Sigma$, we conclude from (2.18) that

$$
\left(\nabla_{y} w(t)(\pi(t)) \mid \pi^{\prime}(t)\right)=R(w, r, \rho)(\pi(t)), \quad t \in I_{\varepsilon} .
$$

Hence we see that

$$
\partial_{t} u\left(t, x_{0}\right)=\left[\Theta_{*}^{\rho(t)} w^{\prime}(t)\right]\left(x_{0}\right)+\left[\Theta_{*}^{\rho(t)} R(w, r, \rho)\right]\left(x_{0}\right), \quad t \in I_{\varepsilon} .
$$

Using $w^{\prime}+A(\rho) w=R(w, r, \rho)-w$ on $\dot{J} \times D$, we find

$$
\begin{aligned}
\Theta_{*}^{\rho(t)} w^{\prime}(t) & =-\Theta_{*}^{\rho(t)} A(\rho) w-\Theta_{*}^{\rho(t)} w-\Theta_{*}^{\rho(t)} R(w, r, \rho) \\
& =\Delta u(t)-u(t)-\Theta_{*}^{\rho(t)} R(w, r, \rho),
\end{aligned}
$$

and therefore

$$
\partial_{t} u\left(t_{0}, x_{0}\right)=\Delta u\left(t_{0}, x_{0}\right)-u\left(t_{0}, x_{0}\right) .
$$

The verifications of (1.1) and (1.3)-(1.6) can be done analogously (and are even easier than that of (1.2)). 


\section{The reduced system}

In this section we reduce system (2.15) by eliminating the transformed pressure $r$. In order to do this, we need a suitable representation of the solution operator to the boundary value problem

$$
A(\rho) r=g \quad \text { in } D, \quad r=h \quad \text { on } \Sigma .
$$

Given $q \in(1, \infty), l \in[1, \infty]$, and $s \geqslant 0$, we write $H_{q}^{s}$ and $B_{l l}^{s}$ for the usual Bessel potential and Besov spaces, respectively (cf. [21]). Recall that $H_{q}^{k}$ coincides in case $k \in \mathbb{N}$ with the usual Sobolev space $W_{q}^{k}$ of order $k$ built over $L_{q}$ and that $B_{\infty \infty}^{s}=B U C^{s}$, provided $s$ is not a natural number. Here, $B U C^{s}$ stands, in the case $s \in(0,1)$, for the Banach space of all bounded and uniformly Hölder continuous functions of exponent $s$. If $s=k+s^{\prime}$ for some $k \in \mathbb{N}$ and $s^{\prime} \in(0,1)$ the space $B U C^{s}$ consists of all $k$-times differentiable functions such that all derivatives up to order $k$ belong to $B U C^{s^{\prime}}$.

We now fix $q>n+1$ and choose $\tau \in(0,1-n / q)$ as well as

$$
\varepsilon \in(0, \min \{1-1 / 2 q,(1-n / q-\tau) / 3\})
$$

This choice of $\varepsilon$ is motivated by various embedding properties of function spaces which will be introduced in the next section. Moreover, we use the abbreviation $U:=B_{q q}^{4-3 \varepsilon-1 / q}(\Sigma) \cap$ Ad. Observe that $U$ is contained in $B U C^{3+\tau}(\Sigma)$, by Sobolev's embedding theorem.

LEMMA 3.1 Let $\delta \in[0,1]$. Then:

(a) $A \in C^{\infty}\left(U, \mathcal{L}\left(H_{q}^{2+\delta}(D), H_{q}^{\delta}(D)\right) \cap \mathcal{L}\left(B U C^{2+\delta}(D), B U C^{\delta}(D)\right)\right)$.

(b) $B \in C^{\infty}\left(U, \mathcal{L}\left(H_{q}^{2+\delta}(D), B_{q q}^{1+\delta-1 / q}(\Sigma)\right) \cap \mathcal{L}\left(B U C^{2+\delta}(D), B U C^{1+\delta}(\Sigma)\right)\right)$.

(c) There exists

$$
(S, T) \in C^{\infty}\left(U, \mathcal{L}\left(H_{q}^{\delta}(D) \times B_{q q}^{2+\delta-1 / q}(\Sigma), H_{q}^{2+\delta}(D)\right)\right)
$$

such that, given $\rho \in U$ and $(g, h) \in H_{q}^{\delta}(D) \times B_{q q}^{2+\delta-1 / q}(\Sigma)$, the unique solution $r \in$ $H_{q}^{2+\delta}(D)$ to (3.1) is given by $r=S(\rho) g+T(\rho) h$.

(d) $S \in C^{\infty}\left(U, \mathcal{L}\left(B U C^{\delta}(D), B U C^{2+\delta}(D)\right)\right)$.

Proof. Observe that the coefficients of $A(\rho)$ belong to $B U C^{1+\tau}(D)$ and those of $B(\rho)$ to $B U C^{2+\tau}(\Sigma)$; then the first two assertions follow as in the proof of Lemma 2.2 in [12]. Assertions (c) and (d) are now consequences of (a) and known elliptic regularity theory (cf. Theorem 4.3.3 in [21] and the proof of Lemma 2.3 in [12]).

Using Lemma 3.1 it is easily verified that the pair $(w, \rho)$ satisfies the following reduced system:

$$
\begin{aligned}
\partial_{t} w+A(\rho) w & =\tilde{F}_{1}(w, \rho) & & \text { in } \dot{J} \times D, \\
w & =\chi(\rho) & & \text { on } J \times \Sigma, \\
\partial_{t} \rho+B(\rho) T(\rho) H(\rho) & =B(\rho) S(\rho) g(w, \rho) & & \text { on } \dot{J} \times \Sigma, \\
w(0, \cdot) & =w_{0} & & \text { in } D, \\
\rho(0, \cdot) & =\rho_{0} & & \text { on } \Sigma,
\end{aligned}
$$

where

$$
\begin{aligned}
\tilde{F}_{1}(w, \rho) & :=R(w, r(w, \rho), \rho)-w, \\
r(w, \rho) & :=S(\rho) g(w, \rho)+T(\rho) H(\rho) .
\end{aligned}
$$


Due to the inhomogeneous Dirichlet boundary condition on $w$, system (3.2) is not yet suitable to be treated in the abstract frame presented in the preceding section. To arrive at a homogeneous boundary condition observe that $\chi(\rho)=\psi \circ \Theta_{\rho}$ is well defined on $\mathbb{R}^{n}$. Thus, if we let $v:=w-\chi(\rho)$ and $v_{0}:=w_{0}-\chi\left(\rho_{0}\right)$, then the pair $(v, \rho)$ satisfies the system

$$
\begin{aligned}
\partial_{t} v+A(\rho) v & =\hat{F}_{1}(v, \rho)-A(\rho) \chi(\rho)-Q(v, \rho) & & \text { in } \dot{J} \times D, \\
v & =0 & & \text { on } J \times \Sigma, \\
\partial_{t} \rho+B(\rho) T(\rho) H(\rho) & =-B(\rho) S(\rho) \hat{g}(v, \rho) & & \text { on } \dot{J} \times \Sigma, \\
v(0, \cdot) & =v_{0} & & \text { in } D, \\
\rho(0, \cdot) & =\rho_{0} & & \text { on } \Sigma,
\end{aligned}
$$

where

$$
\begin{aligned}
\hat{F}_{1}(v, \rho) & :=R(v+\chi(\rho), r(v+\chi(\rho), \rho))-v-\chi(\rho), \\
\hat{g}(v, \rho) & :=g(v+\chi(\rho), \rho),
\end{aligned}
$$

and

$$
Q(v, \rho)(y):=[\varphi \circ \Lambda] \cdot[B(\rho) r(v+\chi(\rho), \rho) \circ P] \cdot\left[\left(\Theta_{\rho}^{*} \nabla \psi \mid \mu \circ P\right)\right](y)
$$

if $y \in \mathcal{R}_{D}$, and $Q(v, \rho)(y):=0$ if $y \in D \backslash \mathcal{R}_{D}$. The term $Q(v, \rho)$ arises for the same reason as the term $R$ from (2.13) in the transformed system (2.15): The transformation $\chi(\rho)$ of the original Dirichlet data $\psi$ depends on the time variable and we have

$$
\frac{\mathrm{d}}{\mathrm{d} t} \chi(\rho(t))(y)=Q(v, \rho(t))(y), \quad(t, y) \in \dot{J} \times D .
$$

Next, we need a suitable splitting of the mean curvature operator $H(\rho)$. It is well known that this operator has the structure of a quasilinear operator of second order. Indeed, using local coordinates on $\Sigma$ it is possible to show that there is a quasilinear operator $P$ of second order and a nonlinear first order operator $K$ such that $H(\rho)=P(\rho) \rho+K(\rho)$ for any $\rho \in C^{2}(\Sigma)$. In the next lemma we state the precise regularity properties of these operators which we need in what follows. For a proof of these results we refer to Lemma 3.1 of [12].

LEMMA 3.2 Given $s \in(0,1 / q)$, there exist

$$
\begin{aligned}
& P \in C^{\infty}\left(U, \mathcal{L}\left(B_{q q}^{4+s-1 / q}(\Sigma), B_{q q}^{2+s-1 / q}(\Sigma)\right)\right), \\
& K \in C^{\infty}\left(U, B U C^{3-3 \varepsilon-1 / q}(\Sigma)\right)
\end{aligned}
$$

such that $H(\rho)=P(\rho) \rho+K(\rho)$ for any $\rho \in U \cap B_{p p}^{4+s-1 / p}(\Sigma)$.

We are now prepared to introduce the following nonlinear operator $F=\left(F_{1}, F_{2}\right)$ for the pair $Z:=(v, \rho)$ :

$$
\begin{aligned}
& F_{1}(Z):=\hat{F}_{1}(v, \rho)-A(\rho) \chi(\rho)-Q(v, \rho), \\
& F_{2}(Z):=-B(\rho)[T(\rho) K(\rho)+S(\rho) \hat{g}(v, \rho)],
\end{aligned}
$$

as well as the quasilinear operator

$$
\mathbb{A}(Z) Z:=(A(\rho) v, B(\rho) T(\rho) P(\rho) \rho) .
$$

Observe that for fixed $Z_{1}$, the mapping $Z \mapsto \mathbb{A}\left(Z_{1}\right) Z$ is linear. 
Using these operators, we can express the system (3.3) in the form

$$
Z^{\prime}+\mathbb{A}(Z) Z=F(Z), \quad Z(0)=Z_{0},
$$

where, of course, $Z_{0}:=\left(v_{0}, \rho_{0}\right)$.

We call $Z=(v, \rho)$ a classical solution to (3.6) if the following conditions hold:

(i) $Z \in C\left(J, H_{q}^{2}(D) \times U\right) \cap C^{1}\left(J, L_{q}(D) \times B_{q q}^{1-1 / q}(\Sigma)\right)$;

(ii) $v(t) \mid \Sigma=0$ for $t \in J$;

(iii) $Z \in C^{\infty}\left(\dot{J}, H_{q}^{2+k}(D) \times B_{q q}^{4+k-1 / q}(\Sigma)\right)$ for all $k \in \mathbb{N}$;

(iv) $Z$ satisfies (3.6) pointwise on $J$.

LEMMA $3.3 \quad$ (a) Let $Z_{0}=\left(v_{0}, \rho_{0}\right) \in H_{q}^{2}(D) \times U$ with $v_{0} \mid \Sigma=0$ be given, and assume that $Z=(v, \rho)$ is a classical solution to (3.6). Then $(w, r, \rho)$ with $w:=v+\chi(\rho)$ and $r:=S(\rho) g(w, \rho)+T(\rho) H(\rho)$ is a classical solution to (2.15).

(b) If $(w, r, \rho)$ is a classical solution to (2.15) with initial data $\left(w_{0}, \rho_{0}\right) \in H_{q}^{2}(D) \times U$ then $Z:=(w-\chi(\rho), \rho)$ is a classical solution to (3.6) with the corresponding initial value $Z_{0}:=\left(w_{0}-\chi\left(\rho_{0}\right), \rho_{0}\right)$.

Proof. Due to the derivation of (3.6), the statements are easily verified.

\section{The abstract setting and the proof of the main result}

In this section we introduce the abstract frame in which the equation (3.6) will be solved. First we pick $q>n+1$ and $s \in(0,1 / 3 q)$ and keep these numbers fixed hereafter. Using the notation introduced at the beginning of the previous section, we know from the trace theorem that $v \mapsto$ $v \mid \Sigma$ is a retraction from $H_{q}^{\sigma}(D)$ onto $B_{q q}^{\sigma-1 / q}(\Sigma)$, provided $\sigma>1 / q$ (cf. Theorem 3.3.3 in [21]). In particular,

$$
H_{q, 0}^{\sigma}(D):=\left\{v \in H_{q}^{\sigma}(D) ; v \mid \Sigma=0\right\}, \quad \sigma>1 / q,
$$

is a closed subspace of $H_{q}^{\sigma}(D)$.

We now define

$$
E_{0}:=H_{q}^{2 s}(D) \times B_{q q}^{1+3 s-1 / q}(\Sigma), \quad E_{1}:=H_{q, 0}^{2+2 s}(D) \times B_{q q}^{4+3 s-1 / q}(\Sigma) .
$$

Equation (3.6) will be considered in the space $E_{0}$, whereas $E_{1}$ will serve as the domain of the leading linear operator contained in (3.6). We shall also need the complex interpolation spaces $E_{\theta}:=\left[E_{0}, E_{1}\right]_{\theta}$, with $\theta \in(0,1)$, where $[\cdot, \cdot]_{\theta}$ stands for the complex interpolation method (cf. [21]). The next result characterizes most of the spaces $E_{\theta}$.

LEMMA 4.1 Given $\theta \in(0,1)$, we have

$$
E_{\theta}= \begin{cases}H_{q}^{2(\theta+s)}(D) \times B_{q q}^{3(\theta+s)+1-1 / q}(\Sigma) & \text { if } \theta+s<1 / 2 q, \\ H_{q, 0}^{2(\theta+s)}(D) \times B_{q q}^{3(\theta+s)+1-1 / q}(\Sigma) & \text { if } \theta+s>1 / 2 q .\end{cases}
$$


Proof. It follows from Theorem 4.3.3(a) in [21] that

$$
\left[L_{q}(D), H_{q, 0}^{3}(D)\right]_{\eta}= \begin{cases}H_{q}^{3 \eta}(D) & \text { if } 3 \eta<1 / q \\ H_{q, 0}^{3 \eta}(D) & \text { if } 3 \eta>1 / q\end{cases}
$$

Hence the reiteration theorem for the complex interpolation functor yields

$$
\left[H_{q}^{2 s}(D), H_{q, 0}^{2+2 s}(D)\right]_{\theta}= \begin{cases}H_{q}^{2(\theta+s)}(D) & \text { if } \theta+s<1 / 2 q \\ H_{q, 0}^{2(\theta+s)}(D) & \text { if } \theta+s>1 / 2 q\end{cases}
$$

The assertion now follows from Proposition I.2.3.3 in [3], since Besov spaces over a smooth closed manifold are stable under complex interpolation (cf. Theorem 7.4.3 in [22] and Theorem 2.4.7 in [21]).

We also need the following embeddings:

LEMMA 4.2 Given $0 \leqslant r_{0}<r_{1}$, we have

(a) $B U C^{r_{1}}(D) \hookrightarrow H_{q}^{r_{0}}(D)$;

(b) $B U C^{r_{1}}(\Sigma) \hookrightarrow B_{q q}^{r_{0}}(\Sigma)$.

Proof. (i) Assume that $r_{0} \geqslant 1$ and pick $k \in \mathbb{N}$ such that $r_{0}-k \in[0,1)$. Then it is known that $v$ belongs to $H_{q}^{r_{0}}(D)$ if and only if $\partial^{\alpha} v$ belongs to $H_{q}^{r_{0}-k}(D)$ for any $\alpha \in \mathbb{N}^{n}$ with $|\alpha| \leqslant k$. Hence it suffices to treat the case $0 \leqslant r_{0}<r_{1}<1$.

(ii) Due to the boundedness of $D$ we have

$$
B U C(D) \hookrightarrow L_{q}(D), \quad B U C^{1}(D) \hookrightarrow W_{q}^{1}(D)=H_{q}^{1}(D) .
$$

Furthermore, denoting the real interpolation method by $(\cdot, \cdot)_{\theta, q}$, where $\theta \in(0,1)$ and $q \in[1, \infty]$, we have

$$
B U C^{r_{1}}(D)=\left(B U C(D), B U C^{1}(D)\right)_{r_{1}, \infty},
$$

since $r_{1} \in(0,1)$ (cf. the proof of Theorem 2.5.7 in [21]). We now conclude from Theorem 2.4.2 in [21] that

$$
B U C^{r_{1}}(D)=\left(B U C(D), B U C^{1}(D)\right)_{r_{1}, \infty} \hookrightarrow\left(L_{q}(D), H_{q}^{1}(D)\right)_{r_{1}, \infty}=B_{q \infty}^{r_{1}}(D) .
$$

Using Proposition 2.3.2.2 of [21] and the fact that $r_{1}>r_{0}$ we obtain

$$
B U C^{r_{1}}(D) \hookrightarrow B_{q \infty}^{r_{1}}(D) \hookrightarrow B_{q 1}^{r_{0}}(D) \hookrightarrow H_{q}^{r_{0}}(D) .
$$

(iii) Similarly, we have $B U C^{r_{1}}(\Sigma)=\left(B U C(\Sigma), B U C^{1}(\Sigma)\right)_{r_{1}, \infty}$ and thus

$$
B U C^{r_{1}}(\Sigma) \hookrightarrow\left(L_{q}(\Sigma), H_{q}^{1}(\Sigma)\right)_{r_{1}, \infty}=B_{q \infty}^{r_{1}}(\Sigma) \hookrightarrow B_{q q}^{r_{0}}(\Sigma)
$$

for $0 \leqslant r_{0}<r_{1}<1$. The general case is now treated as in (i).

Letting $\alpha:=1-s$, by Lemma 4.1 we have

$$
E_{\alpha}=H_{q, 0}^{2}(D) \times B_{q q}^{4-1 / q}(\Sigma) .
$$


Further, by the choice of $q$ and $s$ it follows that

$$
2 s<\frac{2}{3 q}<\frac{1}{q}<1-\frac{n}{q} .
$$

We now choose

$$
\tau \in(2 s, 1-n / q) .
$$

Then $s<\tau / 2$. Moreover, $s<1 / 3 q<1 / 3 q+\tau / 3$. Thus there is a $\gamma>0$ such that

$$
2(\gamma+s)<\tau, \quad 3(\gamma+s)<\tau+1 / q .
$$

Consequently, Lemma 4.2 implies that

$$
B U C^{\tau}(D) \hookrightarrow H_{q}^{2(\gamma+s)}(D), \quad B U C^{1+\tau}(\Sigma) \hookrightarrow B_{q q}^{3(\gamma+s)+1-1 / q}(\Sigma) .
$$

We now let $\beta:=1-s-\varepsilon$, where $\varepsilon \in(0,1-1 / 2 q)$ will be specified later. By Lemma 4.1, we have

$$
E_{\beta}=H_{q, 0}^{2(1-\varepsilon)}(D) \times B_{q q}^{3(1-\varepsilon)+1-1 / q}(\Sigma) .
$$

Observe further that

$$
\tau<1-n / q, \quad 3(\gamma+s)<\tau+1 / q<1, \quad \tau / 2<1,
$$

by the choice of $\tau$ and $\gamma$. Hence there is an $\varepsilon \in(0,1-1 / 2 q)$ such that

$$
1-3 \varepsilon-n / q>\tau, \quad 1-3 \varepsilon>3(\gamma+s), \quad \tau / 2+\varepsilon<1 .
$$

The first inequality in (4.3) and the generalized Sobolev embedding theorem (cf. Theorem 3.3.1 in [21]) now yield

$$
H_{q}^{1-2 \varepsilon}(D) \hookrightarrow B U C^{\tau}(D), \quad B_{q q}^{1-3 \varepsilon-1 / q}(\Sigma) \hookrightarrow B U C^{\tau}(\Sigma),
$$

while the second inequality in (4.3) implies

$$
B_{q q}^{2-3 \varepsilon-1 / q}(\Sigma) \hookrightarrow B_{q q}^{3(\gamma+s)+1-1 / q}(\Sigma) .
$$

The embeddings (4.4) and (4.5) will be used later in order to verify the mapping properties of the nonlinearity $F$, formally introduced in (3.4). Before giving the full details of these facts, let us mention that

$$
0<\gamma<\beta<\alpha<1 .
$$

Indeed, since $\gamma<\tau / 2-s$, the third inequality in (4.3) gives

$$
\gamma<\tau / 2-s<1-s-\varepsilon=\beta .
$$

The remaining inequalities in (4.6) are clear.

For the sake of simplicity, we write $E_{\theta}^{j}$ for the $j$-th factor of $E_{\theta}$, where $j=1,2$ and $\theta \in[0,1]$. This means e.g. that

$$
E_{1}^{1}=H_{q, 0}^{2+2 s}(D), \quad E_{\alpha}^{2}=B_{q q}^{4-1 / q}(\Sigma)
$$


Furthermore, given $\theta \in[\beta, 1]$, let

$$
X_{\theta}:=\left\{Z=(v, \rho) \in E_{\theta} ; \rho \in \operatorname{Ad}\right\}
$$

Obviously, $X_{\theta}$ is open in $E_{\theta}$. Again, $X_{\theta}^{j}$ stands for the $j$-th factor of $X_{\theta}$ where $j=1,2$. Thus

$$
X_{\alpha}^{1}=E_{\alpha}^{1}=H_{q, 0}^{2}(D), \quad X_{\beta}^{2}=B_{q q}^{4-3 \varepsilon-1 / q}(\Sigma) \cap \operatorname{Ad} .
$$

Observe further that $X_{\beta}^{2}$ coincides with the set $U$ introduced in Section 3. Given $\rho \in X_{\beta}^{2}$, we define a linear operator by

$$
A(\rho): \operatorname{dom}(A(\rho))=: E_{1}^{1}=H_{q, 0}^{2+2 s}(D) \rightarrow H_{q}^{2 s}(D)=E_{0}^{1}, \quad v \mapsto A(\rho) v .
$$

In the following we shall see that $-A(\rho)$ generates a strongly continuous analytic semigroup in $\mathcal{L}\left(E_{0}^{1}\right)$. Since we also need suitable regularity properties of the nonlinear mapping $\rho \mapsto A(\rho)$, let us introduce the following notation. Given two Banach spaces $B_{1}$ and $B_{0}$ with $B_{1} \hookrightarrow B_{0}$ and $B_{1}$ dense in $B_{0}$, let $\mathcal{H}\left(B_{1}, B_{0}\right)$ be the set of all linear operators $A$ in $B_{0}$ such that $\operatorname{dom}(A)=B_{1}$ and $-A$ generates a strongly continuous analytic semigroup in $\mathcal{L}\left(B_{0}\right)$. It follows from the closed graph theorem that $\mathcal{H}\left(B_{1}, B_{0}\right)$ is a subset of $\mathcal{L}\left(B_{1}, B_{0}\right)$ and Theorem I.1.3.1 in [3] shows that $\mathcal{H}\left(B_{1}, B_{0}\right)$ is in fact open in $\mathcal{L}\left(B_{1}, B_{0}\right)$.

LEMMA $4.3 A \in C^{\infty}\left(X_{\beta}^{2}, \mathcal{H}\left(E_{1}^{1}, E_{0}^{1}\right)\right)$.

Proof. (i) It follows easily from (4.4) and Lemma 3.1 that $\rho \mapsto A(\rho)$ maps $X_{\beta}^{2}$ smoothly in $\mathcal{L}\left(E_{1}^{1}, E_{0}^{1}\right)$.

(ii) Fix $\rho \in X_{\beta}^{2}$, and define

$$
A_{0}(\rho): \operatorname{dom}\left(A_{0}(\rho)\right):=H_{q, 0}^{2}(D) \rightarrow L_{q}(D), \quad v \mapsto A(\rho) v .
$$

Then it is well known that $A_{0}(\rho) \in \mathcal{H}\left(H_{q, 0}^{2}(D), L_{q}(D)\right)$. Observe that $E_{0}^{1}=H_{q}^{2 s}(D)=$ $\left[L_{q}(D), H_{q, 0}^{2}(D)\right]_{s}$, and write $A_{s}(\rho)$ for the $H_{q}^{2 s}(D)$-realization of $A_{0}(\rho)$. Then it follows from elliptic regularity theory and from the trace theorem that $\operatorname{dom}\left(A_{s}(\rho)\right)=H_{q, 0}^{2+2 s}(D)=E_{1}^{1}$ and $A_{s}(\rho)=A(\rho)$. Thus interpolation implies that $A(\rho) \in \mathcal{H}\left(E_{1}^{1}, E_{0}^{1}\right)$ (cf. Theorem V.2.1.3. in [3]).

We also need the following generation result for the leading part of the second component of (3.5). LemMA $4.4[\rho \mapsto B(\rho) T(\rho) P(\rho)] \in C^{\infty}\left(X_{\beta}^{2}, \mathcal{H}\left(E_{1}^{2}, E_{0}^{2}\right)\right)$.

Proof. This follows by obvious modifications of the proof of Theorem 4.1 in [12], where the case $q=\infty$ is treated. Indeed, one only has to replace the estimate (5.7) in [12] by the corresponding one in the spaces

$$
E_{1}^{2}=B_{q q}^{4+3 s-1 / q}(\Sigma), \quad E_{0}^{2}=B_{q q}^{1+3 s-1 / q}(\Sigma) .
$$

Moreover, the arguments in step (v) on page 640 in [12] can be carried over by using Theorem 4.1 of [9].

Given $Z=(v, \rho) \in X_{\beta}$, define the linear operator $\mathbb{A}(Z)$ in $E_{0}$ by $\operatorname{setting} \operatorname{dom}(\mathbb{A}(Z)):=E_{1}$ and

$$
\mathbb{A}(Z) W:=(A(\rho) w, B(\rho) T(\rho) P(\rho) \sigma) \quad \text { for } W=(w, \sigma) \in E_{1} .
$$

Then we have 
Corollary $4.5 \mathbb{A} \in C^{\infty}\left(X_{\beta}, \mathcal{H}\left(E_{1}, E_{0}\right)\right)$.

Proof. This is an immediate consequence of Lemmas 4.3 and 4.4, and the characterization of the set $\mathcal{H}\left(E_{1}, E_{0}\right)$ given in Theorem I.1.2.2 of [3].

Let $\pi \in C^{\infty}(\mathbb{R}, \mathbb{R})$ be given. Then $\pi$ induces so-called Nemytskiu operators $\Pi$ acting on various subspaces of $B U C\left(\mathbb{R}^{n}\right)$ by setting

$$
\Pi(v)(x):=\pi(v(x)), \quad v \in B U C\left(\mathbb{R}^{n}\right), x \in \mathbb{R}^{n} .
$$

In the following we do not distinguish notationally between $\pi$ and $\Pi$. With this convention we introduce the operator $F:=\left(F_{1}, F_{2}\right): X_{\beta} \rightarrow E_{0}$ by setting

$$
\begin{aligned}
& F_{1}(Z):=\hat{F}_{1}(v, \rho)-A(\rho) \chi(\rho)-Q(v, \rho), \\
& F_{2}(Z):=B(\rho)[T(\rho) K(\rho)+S(\rho) \hat{g}(v, \rho)],
\end{aligned}
$$

where $Z:=(v, \rho) \in X_{\beta}$. Recall that $\chi(\rho)=\Theta_{\rho}^{*} \psi$ and $g=\Theta_{\rho}^{*} f \Theta_{*}^{\rho}$. Moreover, we have $\hat{g}(v, \rho)=$ $g(v+\chi(\rho))$ and

$$
\hat{F}_{1}(v, \rho)=R(v+\chi(\rho), S(\rho) \hat{g}(v, \rho)+T(\rho) H(\rho), \rho)-v-\chi(\rho) .
$$

Lemma 4.6 $F \in C^{\infty}\left(X_{\beta}, E_{\gamma}\right)$.

Proof. (i) The mean value theorem implies that $g$ maps $B U C^{1+\tau}(D) \times$ Ad smoothly into $B U C^{1+\tau}(D)$ and that $\chi$ maps $B U C^{3}(\Sigma) \cap$ Ad smoothly into $B U C^{3}(D)$. Using the embeddings in (4.4) we therefore recognize that

$$
\hat{g} \in C^{\infty}\left(X_{\beta}, B U C^{1+\tau}(D)\right) .
$$

(ii) Given $(v, \rho) \in X_{\beta}$, it follows from (4.7) and Lemma 3.1(d) that $S(\rho) g(v+\chi(\rho), \rho)$ belongs to $B U C^{2+\tau}(D)$, and hence, using Lemma 3.1(d) again, we get

$$
[(v, \rho) \mapsto S(\rho) g(v+\chi(\rho), \rho)] \in C^{\infty}\left(X_{\beta}, B U C^{2+\tau}(D)\right) .
$$

Invoking Lemma 3.1(b), we conclude that

$$
[(v, \rho) \mapsto B(\rho) S(\rho) g(v+\chi(\rho), \rho)] \in C^{\infty}\left(X_{\beta}, B U C^{1+\tau}(\Sigma)\right) .
$$

Moreover, from Lemmas 3.1 and 3.2 we know that

$$
[\rho \mapsto B(\rho) T(\rho) K(\rho)] \in C^{\infty}\left(X_{\beta}^{2}, B_{q q}^{2-3 \varepsilon-1 / q}(\Sigma)\right) .
$$

Hence (4.1) and (4.5) yield

$$
F_{2} \in C^{\infty}\left(X_{\beta}, E_{\gamma}^{2}\right)
$$

(iii) Given $(h, k) \in B U C^{\tau}(\Sigma) \times B U C^{\tau}(D)$, we define a mapping $r_{0}(h, k): D \rightarrow \mathbb{R}$ by

$$
r_{0}(h, k)(y):= \begin{cases}\varphi(\Lambda(y)) h(P(y)) k(y) & \text { if } y \in \mathcal{R}_{D}, \\ 0 & \text { if } y \in D \backslash \mathcal{R}_{D} .\end{cases}
$$


Recalling that $(P, \Lambda) \in C^{\infty}\left(\mathcal{R}_{D}, \Sigma \times(-a, a)\right)$ and that $\varphi(\xi)=0$ if $|\xi|>3 b$, one easily verifies that $r_{0}(h, k)$ belongs to $B U C^{\tau}(D)$. Moreover, there is a constant $c=c(P, \Lambda)$, independent of $(h, k)$, such that

$$
\left\|r_{0}(h, k)\right\|_{B U C^{\tau}(D)} \leqslant c\|h\|_{B U C^{\tau}(\Sigma)}\|k\|_{B U C^{\tau}(D)} .
$$

This shows that $r_{0}$ is a bounded bilinear mapping from $B U C^{\tau}(\Sigma) \times B U C^{\tau}(D)$ to $B U C^{\tau}(D)$.

(iv) Given $Z:=(v, \rho) \in X_{\beta}$ and $j \in\{1, \ldots, n\}$, let

$$
\begin{aligned}
h^{j}(Z) & :=\left(B(\rho)[T(\rho) H(\rho)+S(\rho) g(v+\chi(\rho), \rho)] \cdot \mu^{j}\right) \circ P, \\
k^{j}(Z) & :=\Theta_{\rho}^{*}\left(\partial_{y^{j}} \Theta_{*}^{\rho}(v+\chi(\rho))\right),
\end{aligned}
$$

where $\mu^{j}$ and $y^{j}$ stand for the $j$-th components of $\mu$ and $y$, respectively. We know from (ii) that $h^{j} \in$ $C^{\infty}\left(X_{\beta}, B U C^{\tau}(\Sigma)\right)$, and one shows as in Lemma 3.1 that $k^{j} \in C^{\infty}\left(X_{\beta}, B U C^{\tau}(D)\right)$. Moreover, observe that

$$
R(v+d, S(\rho) g(v+d, \rho)+T(\rho) H(\rho), \rho)=\sum_{j=1}^{n} r\left(h^{j}(Z), k^{j}(Z)\right),
$$

where $Z=(v, \rho)$. Hence it follows from (4.8), (4.1) and Lemma 3.1 that $\hat{F}_{1} \in C^{\infty}\left(X_{\beta}, E_{\gamma}^{1}\right)$.

(v) The operator $Q$ has the same structure as $R$ (cf. (2.13)). Hence using verbatim the same arguments as in step (iv) we conclude that $Q \in C^{\infty}\left(X_{\beta}, E_{\gamma}^{1}\right)$.

Invoking Lemma 3.1 again, it follows from $\chi \in C^{\infty}\left(X_{\beta}^{2}, B U C^{3}(D)\right)$ (see step (i)) that

$$
\left.[\rho \mapsto A(\rho) \chi(\rho)] \in C^{\infty}\left(X_{\beta}^{2}, B U C^{1}(D)\right)\right]
$$

Combining finally (4.8), step (v) and (4.1) we infer that $F_{1} \in C^{\infty}\left(X_{\beta}, E_{\gamma}^{1}\right)$, which completes the proof.

Proof of Theorem 1. (i) Let $\Omega_{0}$ be a bounded domain of class $B_{q q}^{4-1 / q}$ and choose $\Sigma$ as in Section 2. By assumption there is a $\rho_{0} \in B_{q q}^{4-1 / q}(\Sigma) \cap \mathrm{Ad}=X_{\alpha}^{2}$ such that $\Gamma_{\rho_{0}}=\Gamma_{0}$, where $\Gamma_{0}$ denotes the boundary of $\Omega_{0}$. Let further $\psi \in B U C^{\infty}\left(\mathbb{R}^{n}, \mathbb{R}\right)$ and assume that $u_{0} \in H_{q}^{2}\left(\Omega_{0}\right)$ satisfies the condition $u_{0}-\psi \in H_{q, 0}^{2}\left(\Omega_{0}\right)$. Setting $Z_{0}:=\left(u_{0}-\psi, \rho_{0}\right)$, we see that $Z_{0} \in X_{\alpha}$. Thus Corollary 4.5 and Lemma 4.6 guarantee that we can apply Theorem 12.1 of [2] to get a $t^{+}>0$ and a unique solution

$$
Z=(v, \rho) \in C\left(\left[0, t^{+}\right), X_{\alpha}\right) \cap C^{1}\left(\left(0, t^{+}\right), E_{0}\right) \cap C\left(\left(0, t^{+}\right), E_{1}\right)
$$

of (3.6). Set

$$
\begin{aligned}
u & :=\Phi_{*}^{\rho}(v+\chi(\rho))=\Phi_{*}^{\rho} v-\psi, \\
p & :=\Phi_{*}^{\rho}(S(\rho) g(v+\chi(\rho), \rho)+T(\rho) H(\rho)), \\
\Gamma(t) & :=\Gamma_{\rho(t)}, \quad t \in\left[0, t^{+}\right) .
\end{aligned}
$$

It follows from Lemmas 3.3 and 2.1 that $(u, p, \Gamma)$ is a classical solution on $J:=\left[0, t^{+}\right)$to $(1.1)-$ (1.6) with initial data $\left(u_{0}, \Gamma_{0}\right)$ and boundary value $\psi$, provided we can show that

$$
v \in C^{\infty}\left(\left(0, t^{+}\right) \times \bar{D}, \mathbb{R}\right), \quad \rho \in C^{\infty}\left(\left(0, t^{+}\right) \times \Sigma, \mathbb{R}\right) .
$$


(ii) In order to verify (4.9), we first observe that the following temporal regularity is provided by the abstract theory:

$$
Z=(v, \rho) \in C^{\infty}\left(\left(0, t^{+}\right), E_{1}\right)
$$

(see Theorem 11.3 in [1]). Due to the Sobolev embeddings (4.4) we therefore obtain in particular

$$
v(\cdot, x), \rho(\cdot, y) \in C^{\infty}\left(\left(0, t^{+}\right), \mathbb{R}\right) \quad \text { for all } x \in \bar{D} \text { and } y \in \Sigma .
$$

(iii) Define

$$
f_{1}(t, x):=F_{1}(v, \rho)(t, x) \quad \text { for }(t, x) \in\left(0, t^{+}\right) \times \bar{D} .
$$

From (4.10) and the proof of Lemma 4.6 we know that $f_{1} \in C^{\tau}\left(\left(0, t^{+}\right) \times \bar{D}, \mathbb{R}\right)$. Moreover, we set $\tilde{A}(t):=A(\rho(t))$ for $t \in\left[0, t^{+}\right)$. By (4.10) and (4.4) the coefficients of $\tilde{A}$ belong to $C^{1+\tau}\left(\left(0, t^{+}\right)\right.$ $\times \bar{D})$. Let $J^{\prime}$ be any compact subinterval of $\left(0, t^{+}\right)$and pick $\eta \in \mathcal{D}(\mathbb{R})$ with $\eta \mid J^{\prime}=0$ and $\operatorname{supp}(\eta)$ $\subset\left(0, t^{+}\right)$. Then $\tilde{G}_{1}:=\eta f_{1}+\eta^{\prime} v \in C^{\tau}\left(\left(0, t^{+}\right) \times \bar{D}, \mathbb{R}\right)$ and $\eta v$ is a solution to the linear parabolic initial boundary value problem

$$
\begin{aligned}
\partial_{t} u+\tilde{A}(t) u & =\tilde{G}_{1} & & \text { in }\left(0, t^{+}\right) \times D, \\
u & =0 & & \text { on }\left[0, t^{+}\right) \times \Sigma, \\
u(0, \cdot) & =0 & & \text { in } D .
\end{aligned}
$$

Thus classical parabolic regularity theory implies that

$$
v \in C\left(\left(0, t^{+}\right), B U C^{2+\tau}(D)\right) \cap C^{1+\tau / 2,2+\tau}\left(\left(0, t^{+}\right) \times \bar{D}\right)
$$

(cf. Theorem IV.5.1 in [18]). Using this and (4.10) one verifies that $f_{1} \in C^{1+\tau}\left(\left(0, t^{+}\right) \times \bar{D}\right)$. Hence $\tilde{G}_{1}$ has the same regularity and since the coefficients of $\tilde{A}$ belong to $C^{1+\tau}\left(\left(0, t^{+}\right) \times \bar{D}\right)$ we see from [18, Theorem IV.5.1] that

$$
v \in C\left(\left(0, t^{+}\right), B U C^{3+\tau}(D)\right) \cap C^{(3+\tau) / 2,3+\tau}\left(\left(0, t^{+}\right) \times \bar{D}\right) .
$$

At this point the bootstrapping argument for $v$ cannot be repeated since we can only guarantee that the coefficients of $\tilde{A}$ belong to $C^{1+\tau}\left(\left(0, t^{+}\right) \times \bar{D}\right)$.

(iv) Define

$$
f_{2}(t):=B(\rho(t)) S(\rho(t)) \hat{g}(v(t), \rho(t)), \quad t \in\left(0, t^{+}\right) .
$$

Then (4.10) and (4.1) imply, as in Lemma 3.1, that

$$
f_{2} \in C\left(\left(0, t^{+}\right), B_{q q}^{3(\gamma+s)+3-1 / q}(\Sigma)\right) .
$$

Let now $t_{1} \in\left(0, t^{+}\right)$and set $\rho_{1}:=\rho\left(t_{1}\right) \in B_{q q}^{4+3 s-1 / q}(\Sigma)$. We take $\rho_{1}$ as initial value for the abstract evolution equation

$$
\partial_{t} \rho+B(\rho) T(\rho) P(\rho) \rho=\tilde{F}_{2}(\rho)+f_{2}(t), \quad \rho\left(t_{1}\right)=\rho_{1},
$$

where, of course, $\tilde{F}_{2}(\rho)=B(\rho) T(\rho) K(\rho)$. Arguing as in [12, p. 635], we find that $\rho \in$ $C\left(\left[t_{1}, t^{+}\right), B_{q q}^{4+3 s-1 / q}(\Sigma)\right)$. This bootstrapping procedure can be repeated and after a finite number of steps we obtain

$$
\rho \in C\left(\left(0, t^{+}\right), B_{q q}^{6-1 / q}(\Sigma)\right) .
$$


At this point the spatial regularity of $\rho$ cannot be improved, due to (4.12). Nevertheless (4.13) and (4.10) make it possible to repeat step (iii) to get

$$
v \in C\left(\left(0, t^{+}\right), B U C^{4+\tau}(D)\right) \cap C^{(4+\tau) / 2,4+\tau}\left(\left(0, t^{+}\right) \times \bar{D}\right) .
$$

Using this, as in step (iv) we obtain

$$
\rho \in C\left(\left(0, t^{+}\right), B_{q q}^{8-1 / q}(\Sigma)\right) .
$$

By induction we now conclude that

$$
\begin{aligned}
& v \in C\left(\left(0, t^{+}\right), B U C^{k+\tau}(D)\right) \cap C^{(k+\tau) / 2, k+\tau}\left(\left(0, t^{+}\right) \times \bar{D}\right), \\
& \rho \in C\left(\left(0, t^{+}\right), B_{q q}^{k-1 / q}(\Sigma)\right) \cap C^{\infty}\left(\left(0, t^{+}\right), B_{q q}^{4-1 / q}(\Sigma)\right)
\end{aligned}
$$

for any $k \in \mathbb{N}$. This implies (4.9).

\section{REFERENCES}

1. Amann, H. Dynamic theory of quasilinear parabolic equations-I. Abstract evolution equations. Nonlinear Anal. 12 (1988), 895-919. Zbl 0666.35043 MR 89j:35072

2. Amann, H. Nonhomogeneous linear and quasilinear elliptic and parabolic boundary value problems. Function Spaces, Differential Operators and Nonlinear Analysis, H. J. Schmeisser and H. Triebel (eds.), Teubner, Stuttgart (1993), 9-126. Zbl 0810.35037 MR 94m:35153

3. Amann, H. Linear and Quasilinear Parabolic Problems, Volume I: Abstract Linear Theory. Birkhäuser, Basel (1995). Zbl 0819.35001 MR 96g:34088

4. BAZALIY, B. V. Stefan problem for the Laplace equation taking into account the curvature of the free boundary. Ukrainian Math. J. 49 (1997), 1465-1484.

5. BAZALIY, B. V. \& Friedman, A. A free boundary problem for an elliptic-parabolic system: application to a model of tumor growth. Comm. Partial Differential Equations 28 (2003), 517-560. Zbl pre01912709 MR 2004c:35420

6. BYRne, H. M. \& Chaplain, M. A. J. Growth of nonnecrotic tumors in the presence and absence of inhibitors. Math. Biosci. 130 (1995), 151-181. Zbl 0836.92011

7. Byrne H. M. \& Chaplain, M. A. J. Growth of necrotic tumors in the presence and absence of inhibitors. Math. Biosci. 135 (1996), 187-216. Zbl 0856.92010

8. Chen, X., Hong, J., \& YI, F. Existence, uniqueness and regularity of classical solutions of the Mullins-Sekerka problem. Comm. Partial Differential Equations 21 (1997), 1705-1727. Z Zbl 0884.35177 MR 97h:35238

9. Escher, J. The Dirichlet-Neumann operator on continuous functions, Ann. Scuola Norm. Sup Pisa 21 (1994), 235-266. Zbl 0810.35017 MR 95g:35045

10. EsCHER, J. On moving boundaries in elliptic-parabolic systems. Evolution Equations: Applications to Physics, Industry, Life Sciences, and Economics, Progr. Nonlinear Differential Equations Appl. 55, Birkhäuser, Basel (2003), 149-156. Zbl pre01996152 MR 2013187

11. Escher, J. \& Simonett, G. On Hele-Shaw models with surface tension. Math. Res. Lett. 3 (1996), 467-474. Zbl 0860.35149 MR 97i:35145

12. Escher, J. \& Simonett, G. Classical solutions for Hele-Shaw models with surface tension, Adv. Differential Equations 2 (1997), 619-642. Zbl 1023.35527 MR 98b:35204

13. Friedman, A. \& Reitich, F. Analysis of a mathematical model for growth of tumor. J. Math. Biol. 38 (1999), 262-284. Zbl 0944.92018 
14. Friedman, A. \& ReItich, F. Symmetry-breaking bifurcation of analytic solutions to free boundary problems: an application to a model of tumor growth, Trans. Amer. Math. Soc. 353 (2001), 1587-1634. Zbl 0983.35019 MR 2002a:35208

15. Friedman, A. \& Reitich, F. Nonlinear stability of quasi-static Stefan problems with surface tension: a continuation approach, Ann. Scuola Norm. Sup. Pisa 30 (2001), 341-403. MR 2003e:35326

16. Greenspan, H. P. Models for the growth of a solid tumor by diffusion. Stud. Appl. Math. 51 (1972), 317-340. Zbl 0257.92001 MR 97k:58047

17. GREENSPAN, H. P. On the growth and stability of cell cultures and solid tumors, J. Theor. Biol. 56 (1976), 229-242. MR 55 \#2183

18. LAdyŽEnsKaja, O. A., Solonnikov, V. A. \& URAL'CEVA, N. N. Linear and Quasilinear Equations of Parabolic Type. Transl. Math. Monogr. 23, Amer. Math. Soc., Providence, RI (1968).

19. LeE, J. L. Riemannian Manifolds. Springer, New York (1995).

20. Lunardi, A. Analytic Semigroups and Optimal Regularity in Parabolic Problems. Birkhäuser, Basel (1995). Zbl 0816.35001 MR 96e:47039

21. Triebel, H. Theory of Function Spaces. Birkhäuser, Basel (1983). Zbl 0546.46027 MR 86j:46026

22. Triebel, H. Theory of Function Spaces II. Birkhäuser, Basel (1992). Zbl 0763.46025 MR 93f:46029 